\title{
Ultra-high bitrate optical phase conjugation/wavelength conversion in DSF and SOA with a novel configuration incorporating inline fibre DFB lasers
}

\author{
S.Y. Set, S. Yamashita, M. Ibsen, R.I. Laming, D., Nesset, A.E. Kelly and C. Gilbertas
}

\begin{abstract}
The authors report a novel polarisation insensitive optical phase conjugator or wavelength converter using orthogonally polarised fibre distributed feedback lasers as four-wave mixing pumps in both dispersion shifted fibre and semiconductor optical amplifiers. Ultra-high bit rate operation up to $40 \mathrm{Gbit} / \mathrm{s}$ and mid-span spectral inversion transmission over $204 \mathrm{~km}$ of standard fibre is demonstrated.
\end{abstract}

Introduction: Dispersion shifted fibre (DSF) and semiconductor optical amplifier (SOA) are two of the most promising media for optical phase conjugation and wavelength conversion using FWM [1,2]. These are key functions in future high bitrate transmission systems for dispersion compensation using MSSI [3,4] and switching in WDM networks. Key issues in FWM are the conversion efficiency, especially the relatively low efficiency in DSF [5], and polarisation independence. The efficiency has been increased by using both novel fibres [6] and SOAs [7]. Polarisation independent FWM has been demonstrated in both SOA [8] and in DSF [9], using two orthogonally polarised pumps. With this technique, 40 Gbit/s $102 \mathrm{~km} \mathrm{MSSI}$ transmission has been demonstrated using SOA-based conjugator [10]. However this configuration results in high complexity and component count.

In this Letter, we report the use of a novel optical phase conjugator configuration using inline fibre distributed feedback (DFB) lasers[11] in 20 and $40 \mathrm{Gbit} / \mathrm{s}$ system experiments.

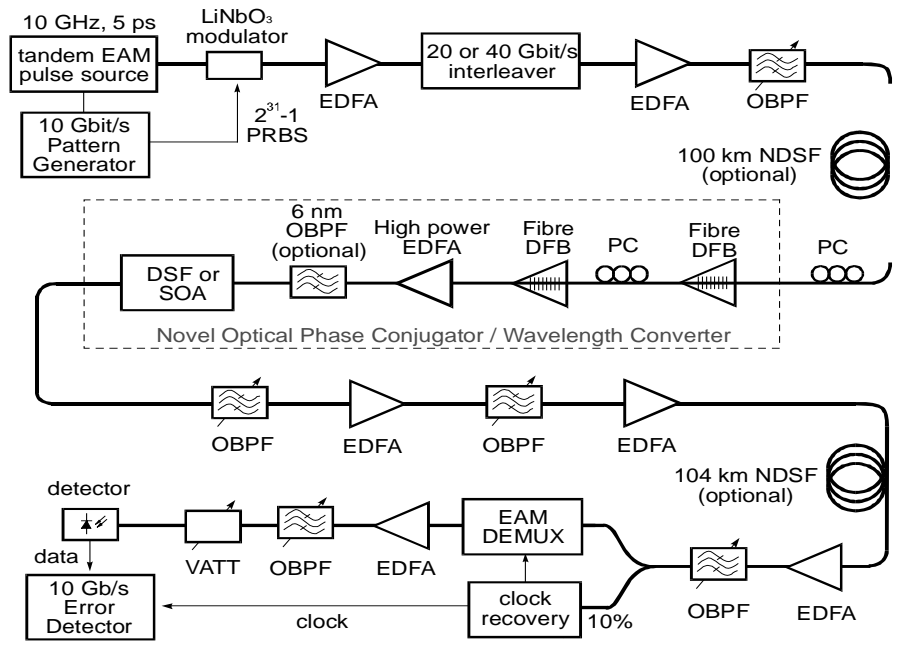

Fig. 1 Experimental setup

Experiment: Fig. 1 shows the experimental setup. The signal source is a $10 \mathrm{GHz}$, transform limited 5 ps pulse train generated using a tandem electroabsorption modulator (EAM) configuration

[10]. It is externally modulated using a lithium niobate $\left(\mathrm{LiNbO}_{3}\right)$ modulator driven by a 10 Gbit/s, $2^{31}-1$ pseudo-random bit sequence (PRBS) and interleaved by a 10 to $20 / 40 \mathrm{Gbit} / \mathrm{s}$ interleaver. We launch the modulated signal directly into two inline cascaded, single polarisation $\mathrm{Er}^{3+}: \mathrm{Yb}^{3+}$ fibre DFB lasers which serve as the FWM pumps at $1551.5 \mathrm{~nm}$ and $1552.8 \mathrm{~nm}$. Each of the fibre DFB lasers is pumped by separate $980 \mathrm{~nm}$ diodes to give flexibility in power balance of the pumps. The 1.3 $\mathrm{nm}$ wavelength detuning of the pumps is near the minimum required for high bitrate operation [9] as in the SOA case, polarisation dependence increases with this detuning [10]. A $23 \mathrm{dBm}$ high power optical amplifier is used to boost the signal and pumps before they are launched into the nonlinear mixing media, which is either 440m of highly nonlinear DSF (HNLDSF) or a $2 \mathrm{~mm}$ long SOA operating at high injection current $550 \mathrm{~mA}$.

The phase conjugate signal generated by FWM is subsequently filtered and amplified by two 1.3 nm polarisation insensitive optical bandpass filters (OBPFs) and two erbium-doped fibre amplifiers (EDFAs). The received signal is demultiplexed by an EAM (DEMUX) to $10 \mathrm{Gbit} / \mathrm{s}$ and the error performance of each channel is measured. 

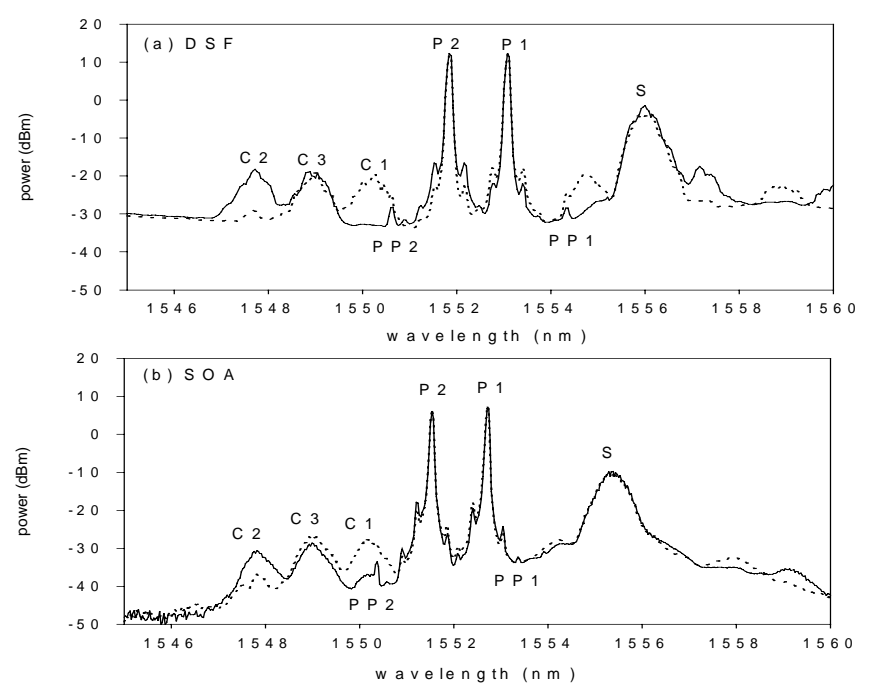

Fig 2 Optical spectra of (a) DSF- and (b) SOA-based conjugators (solid lines and dotted lines represent two extreme signal polarisation cases)

Fig. 2(a) and (b) show the output spectra of the DSF- and SOA-based conjugators respectively. In the SOA case, the signal wavelength is tuned closer to the pumps for higher conversion efficiency and a $6 \mathrm{~nm}$ OBPF is inserted to filter the inline fibre distributed feedback (DFB) lasers out-of-band ASE. The state of polarisations (SOPs) of the two fibre DFB lasers are orthogonally aligned using a polarisation controller (PC) inserted between them. Orthogonal SOPs could be achieved by monitoring the output spectrum of the conjugator and minimise the pump1-pump2 FWM components, PP1 and PP2 (Fig. 2), to 40 dB below the pumps. Spectrally, it can be seen that any changes in signal SOP do not change the conjugate (C3) level significantly, hence polarisation independent operation is demonstrated.
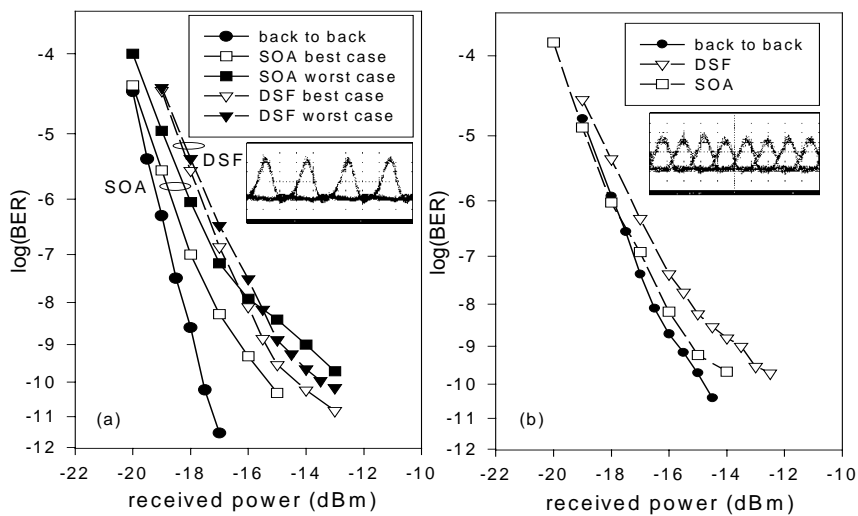

Fig. 3 Bit error rate performance: (a) $20 \mathrm{Gbit} / \mathrm{s}$, polarisation independent and (b) $40 \mathrm{Gbit} / \mathrm{s}$

The BER results of $20 \mathrm{Gbit} / \mathrm{s}$ optical phase conjugation are shown in Fig.3(a). Polarisation sensitivity as low as $0.5 \mathrm{~dB}$ in power penalty is achieved in the DSF-based conjugator. In the SOA, polarisation induced power penalty is measured to be $1.5 \mathrm{~dB}$ due to extra polarisation dependence in the $6 \mathrm{~nm}$ OBPF. We have error free results at a received power of $>-10 \mathrm{dBm}$ despite signal polarisation fluctuations. Fig 3(b) shows $40 \mathrm{Gbit} / \mathrm{s}$ operation with a single fibre DFB laser pump (polarisation sensitive). However, in the polarisation insensitive configuration, we have encountered an error floor at $\sim 10^{-9}$ BER. This is due to the ASE-noise at the conjugate wavelength affecting the conjugate optical signal-to-noise ratio [5]. Despite the error floor, we have carried out MSSI transmission over $204 \mathrm{~km}$ of standard fibre at $40 \mathrm{Gbit} / \mathrm{s}$. The received demultiplexed signal shows good eyes with low polarisation dependency in the SOA-based conjugator (Fig.4) and similar performance is obtained DSF. In order to improve the error performance, one could increase the power of the DFB pumps thus eliminating the need for the amplifier prior to the nonlinear media. This could be done by using a dual-pumped fibre DFB for example. Incorporation of efficient pre-conjugate and post-conjugate filtering will also improve these results [5]. 


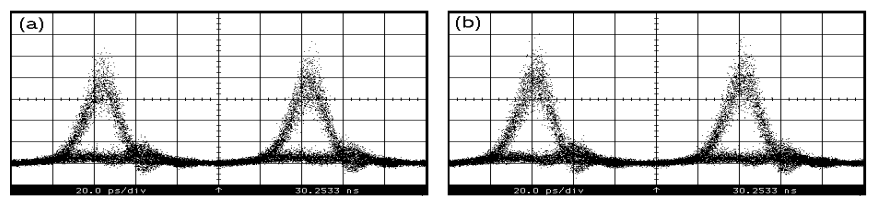

Fig. 4 Eye diagrams of $204 \mathrm{~km}$ MSSI transmission: (a) minimum and (b) maximum received signal

Conclusion: We have demonstrated polarisation insensitive optical phase conjugation and wavelength conversion with a novel in-line fibre DFB laser pump configuration. This configuration has shown good performance both with SOA and DSF nonlinear elements at $20 \mathrm{Gbit} / \mathrm{s}$. With the use of a HNL-DSF or a long SOA, practical polarisation insensitive, wideband phase conjugators are realisable, suitable for future WDM and MSSI applications.

Acknowledgements: The authors would like to thank Dr M. Onishi and Sumitomo Electric Industries, Ltd. for the provision of the HNL-DSF. This work is partially supported by the European Commission through the ACTS projects 'MIDAS' and 'HIGHWAY'. The ORC is an EPSRC funded interdisciplinary research centre.

S. Y. Set, S. Yamashita, M. Ibsen and R. I. Laming (Optoelectronics Research Centre, University of Southampton, Southampton SO17 1BJ, UK)

D. Nesset, A. E. Kelly and C. Gilbertas (BT Laboratories, Martlesham Heath, Ipswich IP5 3RE, UK)

\section{References}

[1] INOUE, K., and TOBA, H.: 'Wavelength conversion experiment using fiber four-wave mixing', IEEE Photon. Technol. Lett., 1992, 4, (1), pp. 69-72

[2] TATHAM, M.C., SHERLOCK G., and WESTBROOK, L.D.: '20-nm optical wavelength conversion using nondegenerate 4-wave- mixing', IEEE Photon. Technol. Lett., 1993, 5, (11), pp. 1303-1306

[3] WATANABE, S., NAITO, T., and CHIKAMA, T.: 'Compensation of chromatic dispersion in a single-mode fiber by opticalphase conjugation', IEEE Photon. Technol. Lett., 1993, 5, (1), pp. 92-95

[4] RØYSET, A., SET, S.Y., GONCHARENKO, I.A., and LAMING, R.I.: 'Linear and nonlinear dispersion compensation of short pulses using midspan spectral inversion', IEEE Photon. Technol. Lett., 1996, 8, (3), pp. 449-451

[5] SET, S.Y., GEIGER, H., LAMING, R.I., COLE, M.J., and REEKIE, L.: 'Optimisation of DSF- and SOA-based phase conjugators by incorporating noise-suppressing fibre gratings', IEEE J. Quantum Electron., 1997, 33,(10), pp. 1694-1698

[6] ONISHI, M., OKUNO, T., KASHIWADA, T., ISHIKAWA, S., AKASAKA, N., and NISHIMURA, M.: 'Highly nonlinear dispersion shifted fiber and its application to broadband wavelength converter', Proc. ECOC'97, Edinburgh, UK, 1997, Vol. 2, pp. 115-118

[7] KELLY, A.E., MARCENAC, D.D., and NESSET, D.: '40 Gbit/s wavelength conversion over $24.6 \mathrm{~nm}$ using FWM in a semiconductor optical amplifier with an optimised MQW active region', Electron. Lett., 1997, 33, (25), pp. 2123-2124

[8] JOPSON, R.M., and TENCH, R.E.: 'Polarisation-independent phase conjugation of lightwave signals', Electron. Lett., 1993, 29, (25), pp.2216-2217

[9] INOUE, K.: 'Polarisation independent wavelength conversion using fibre four-wave mixing with two orthogonal pump lights of different frequencies', J. Lightwave Technol., 1994, 12, (11), pp. 1916-1920

[10] MARCENAC, D.D., NESSET, D., KELLY, A.E., and GAVRILOVIC, D.: '40 Gbit/s transmission over $103 \mathrm{~km}$ of NDSF using polarisation independent mid-span spectral inversion by four-wave mixing in a semiconductor optical amplifier', Electron. Lett., 1998, 34, (1), pp. 100-101

[11] YAMASHITA, S., SET, S.Y., and LAMING, R.I.: “A novel polarisation independent phase conjugator/ wavelength converter utilising inline fibre DFB lasers," submitted to IEEE Photon. Technol. Lett., 1998. 